\title{
Research Paper \\ The Effect of a Period of Kinesiotherapy and Reflexology on Pain Intensity and Range of Motion in Elderly Women With Knee Osteoarthritis
}

\author{
*Fatemeh Kiani Dooghabadi ${ }^{1}$ (1), Nahid Khoshraftar Yazdi ${ }^{2}$ (1), Hossein Farzaneh ${ }^{3}$ (1)
}

1. Department of Sports Pathology and Corrective Movements, Faculty of Human Sciences, Imam Reza International University, Mashhad, Iran 2. Department of Corrective Exercise and Sport Pathology, Faculty of Sport Sciences, Ferdowsi University of Mashhad, Mashhad, Iran.

3. Department of Sports Science, Kashmar Higher Education Institute, Kashmar, Iran.

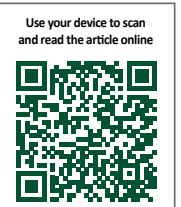

Citation: Kiani Dooghabadi F, Khoshraftar Yazdi N, Farzaneh H. [The Effect of a Period of Therapeutic Exercise and Reflexology on Pain Intensity and Range of Motion in Elderly Women with Knee Osteoarthritis (Persian)]. Journal of Sport Biomechanics 2020; 6(2):98-109. https://doi.org/10.32598/biomechanics.6.2.2

doi'https://doi.org/10.32598/biomechanics.6.2.2

Keywords:

Exercise therapy,

Musculoskeletal manipulation, Pain, Range of motion, Aged, Osteoarthritis, Knee

\begin{abstract}
A B S T R A C T
Objective The aim of this study was to compare the effect of a period of therapeutic exercise and reflexology on pain intensity and range of motion in elderly women with knee osteoarthritis.

Methods 45 elderly women with osteoarthritis of the knee were purposefully selected and randomly divided into three groups of 15: therapeutic exercise, reflexology and control. The therapeutic exercise and reflexology group practiced therapy and reflexology for 4 weeks and 5 sessions per week. The control group also performed their daily activities. To assess the intensity of pain and the range of motion of the subject's knee, the visual pain scale VAS and goniometer were used in pre-test and post-test, respectively. Data analysis was performed by one-way analysis of variance and Tukey's Post Hoc test.

Results The results showed that both types of therapeutic exercise protocol and reflexology have positive and significant effects on reducing pain intensity and increasing range of motion of the knee joint of the subjects $(\mathrm{P} \leq 0.05)$, and the therapeutic exercise protocol compared to the protocol. Reflexology had more significant effects on the rate of increase in range of motion ( $P=0.001 ; P=0.001$, respectively).

Conclusion Based on the results, the use of both protocols of therapeutic exercise and reflexology is recommended to reduce the severity of pain and increase the range of motion of elderly women with osteoarthritis of the knee. Simultaneous use of these two protocols for treatment and greater effectiveness in this group of patients is recommended
\end{abstract}

\section{Extended Abstract}

\section{Introduction}

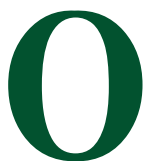

steoarthritis (a degenerative disease of the knee joint) is one of the most common musculoskeletal disorders, which significantly causes motor disabilities and pain in the knee joint $[1,2]$. According to reports, the prevalence of osteoarthritis is between 30\%-40\% among the elderly and $72.6 \%$ among women, which is higher than other age groups [3]. Heavy expenses are spent annually to diagnose, treat, and rehabilitate this disease [8]. The primary treatment for arthritis is pharmacotherapy [7, 9]. However, other non-pharmacological and complementary methods have been considered conservative treatments for these diseases $[10,11]$.

Kinesiotherapy, as an essential method and with the function of improving muscle strength, increasing the depth perception, and strengthening the reflex response, has been introduced to control and prevent this disease's progression

\section{* Corresponding Author:}

Fatemeh Kiani Dooghabadi, MD.

Address: Department of Sports Pathology and Corrective Movements, Faculty of Human Sciences, Imam Reza International University, Mashhad, Iran. Tel: +98 (915) 0464252

E-mail: kiyani.fatemeh20@gmail.com 
[11]. Since the patients with knee osteoarthritis are less likely to exercise due to old age, pain, and disability and they find these activities painful and sometimes risky, complementary and alternative methods (hand and foot reflexology) that do not require actions have been identified as more popular and suitable with these patients $[10,12,13]$. The study aimed to investigate the effect of a course of kinesiotherapy and reflexology on pain intensity; it introduced a range of motion in older women with knee osteoarthritis.

\section{Methods}

In this quasi-experimental study, 45 elderly women with knee osteoarthritis were purposively selected and randomly divided into three groups of 15 named "kinesiotherapy", "reflexology", and "control". The "kinesiotherapy" and "reflexology" groups practiced exercise therapy and reflexology for 4 weeks and 5 sessions per week $[9,19,20]$. The control group performed their daily activities. To assess the severity of pain and the range of motion of the subject's knee, the Visual Analogue Scale (VAS) of pain and goniometer was used in pre-test and post-test, respectively [8]. Data were analyzed by one-way ANOVA and Tukey's Post-Hoc tests.

\section{Results}

The results showed that both kinesiotherapy and reflexology protocols positively and significantly reduce pain intensity and increase the range of motion of the subjects' knee joint $(\mathrm{P} \leq 0.05)$. The kinesiotherapy protocol had more significant effects on the increase in activity range than the reflexology protocol $(\mathrm{P}=0.001, \mathrm{P}=0.001$, respectively). The dependent t-test results regarding the comparison of the "amount of pain" and "range of motion" of the subjects' knees are presented separately in Tables 1 and 2.

As shown in Tables 1 and 2, there is a significant difference between the values of pain and range of motion of both "kinesiotherapy" and "reflexology" groups $(\mathrm{P} \leq 0.05)$. However, these differences are not seen in the control group.

\section{Discussion and Conclusion}

This study aimed to investigate the effect of a course of kinesiotherapy and reflexology on pain intensity and range of motion in elderly women with knee osteoarthritis. In general, this study's results revealed that both the implementation of exercise therapy protocol and reflexology movements had positive and significant effects on reducing

Table 1. Results of the dependent t-test (comparison of the subjects' amount of pain)

\begin{tabular}{cccc}
\hline & \multicolumn{3}{c}{ Mean \pm SD } \\
\cline { 2 - 4 } Groups/Variables & \multicolumn{2}{c}{ The Amount of Pain } & P \\
\cline { 2 - 4 } & Pre-test & Post-test & $0.001^{* *}$ \\
\hline Kinesiotherapy & $20.42 \pm 6.1$ & $92.00 \pm 3.0$ & $0.001^{* *}$ \\
\hline Reflexology & $80.67 \pm 5.0$ & $46.74 \pm 3.0$ & 0.560 \\
\hline Control & $60.82 \pm 5.0$ & $86.91 \pm 5.0$ & $\begin{array}{c}\text { Journal of } \\
\text { Sport Biomechanics }\end{array}$ \\
\hline ** Significant difference at the level of $\mathrm{P} \leq 0.01$. & & \\
\hline
\end{tabular}

Table 2. Results of the dependent t-test (comparison of subjects' range of motion)

\begin{tabular}{cccc}
\hline & \multicolumn{3}{c}{ Mean \pm SD } \\
\cline { 2 - 3 } Groups/Variables & \multicolumn{2}{c}{ The Range of Motion } & P \\
\cline { 2 - 4 } & Pre-test & Post-test & \multirow{2}{*}{$0.001^{* *}$} \\
\hline Kinesiotherapy & $115.2 \pm 35.62$ & $127.3 \pm 19.75$ & $0.21^{*}$ \\
Reflexology & $104.4 \pm 10.15$ & $115.2 \pm 55.18$ & 0.393 \\
\hline Control & $105.3 \pm 56.43$ & $106.2 \pm 00.84$ & Journal of \\
\hline${ }^{*} \mathrm{P} \leq 0.01 ;{ }^{* *} \mathrm{P} \leq 0.05$. & & Sport Biomechanics
\end{tabular}


the severity of pain and increasing joint range of motion in elderly women with knee osteoarthritis $(\mathrm{P} \leq 0.05)$.

Although no specific and purposive study has been conducted to examine a course of kinesiotherapy and reflexology of severe pain and range of motion in elderly women with knee osteoarthritis, in this regard, numerous studies have investigated the effects of such exercises, especially the impact of reflexology movements on the severity of pain in various abnormalities such as low back pain and thigh osteoarthritis, most of which are consistent with the results of the present study $[14,15]$.

In this regard, for example, a study by Khadiga et al. (2019) on 50 women with knee osteoarthritis revealed that 3 weeks of plantar reflexology had significant effects on pain intensity, functional disability, and range of active and inactive knee movement [14]. In their study, Kielar et al. (2017) investigated the effects of plantar reflexology movements on joint pain and disability in patients with hip osteoarthritis. They confirmed that performing 8 reflexology sessions (45 minutes each session) significantly reduced the subjects' pain intensity.

Because any pain, including back pain and knee pain, acts on specific nerve terminals and activates C-Delte-A fibers, the presence of this pain leads to increased secretion of stress hormones, stimulation of the adrenal gland. Stimulation of the sympathetic nervous system, which these factors, in turn, cause high blood pressure, irregular breathing, shortness of breath, increased respiration rate, and increased heart rate [13]. As a result, these cases' improvement can be mentioned in justifying the positive effects of reflexology.

Another factor affecting pain and range of motion in people with osteoarthritis is inflammation and decreased activity and neuromuscular inhibition, which play an essential role in reducing muscle function to determine depth perception, reducing sensory system integrity accurately (including muscle spindles and decreased visual and vestibular system), and changing the sensitivity of cutaneous and articular receptors [17]. According to the researchers in this study, the effectiveness of the therapeutic exercises used is probably due to the direct use of stretching exercises and open kinetic chain exercises of the thighs and knees in the protocol of therapeutic movements, which through neuromuscular inhibition causes atrophy of muscles around the knee and decreased patient mobility [1].

In general, it can be said that based on the results of this study, the use of both protocols of kinesiotherapy and reflexology to reduce pain and increase range of motion in elderly women with knee osteoarthritis is recommended, and simul- taneous use of these two protocols for treatment and greater effectiveness in this group of patients is recommended.

\section{Ethical Considerations}

\section{Compliance with ethical guidelines}

All ethical principles are considered in this article. The participants were informed of the purpose of the research and its implementation stages. They were also assured about the confidentiality of their information. They were free to leave the study whenever they wished, and if desired, the research results would be available to them. This study was conducted to implement research projects at Imam Reza (AS) Non-Profit University.

\section{Funding}

This article was extracted from MA. thesis of first author at the Department of Sports Pathology and Corrective Movements, Faculty of Human Sciences, Imam Reza International University, Mashhad.

\section{Authors' contributions}

All authors were equally contributed in preparing this article.

\section{Conflicts of interest}

The authors declared no conflict of interest. 


\title{
تأثير يك دوره حركت درمانى و رفلكسولوزى بر شدث درد و دامنه حركتى زنان سالمند مبتلا به استئوآرتريتزانو نورن
}

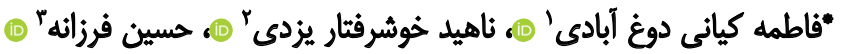

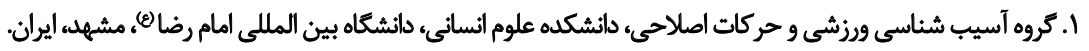

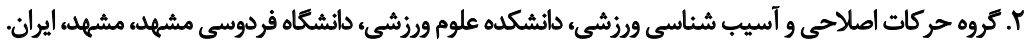 \\ ب. كروه علوم ورزشى، مركز أموزش عالى كاشمر، كاشمر، ايران.
}

هدف همدف از تحقيق حاضر، بررسى تأثير يك دوره حركتدرمائى و رفلكسولوزى برشدت درد و دامنه حركتى زنان سالمند مبتلابه

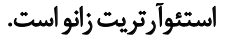

إطلاعاتمقاله: تاريخ دريافت: آI مرداد

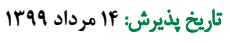

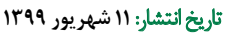

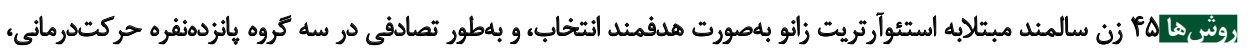

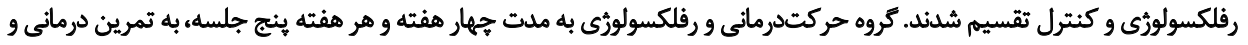

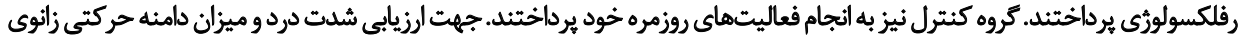

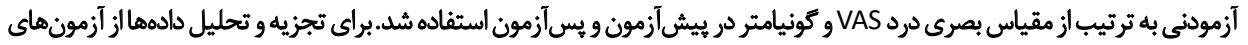

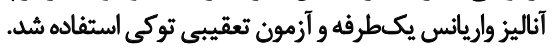

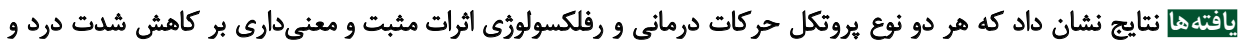

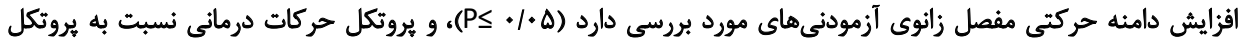

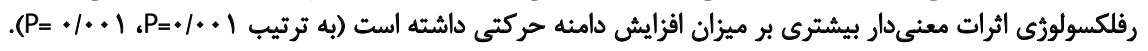

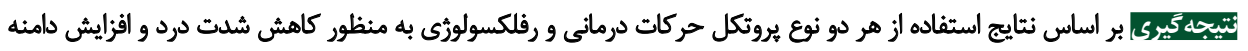

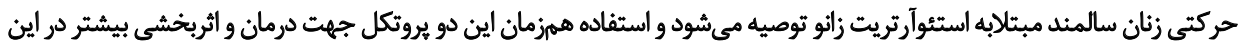

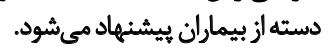

كليدوازٔهها:

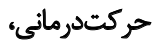

رفلكسولورخى، درد،

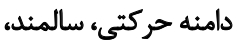

استئوآرتريث زانو

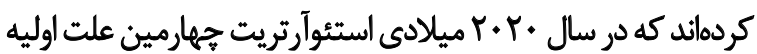

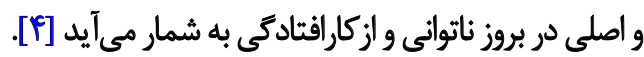

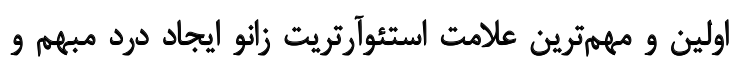

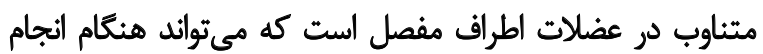

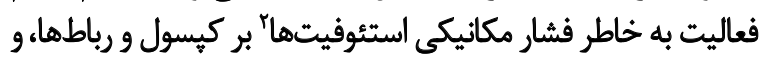

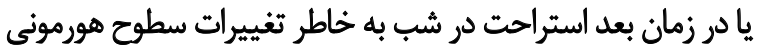

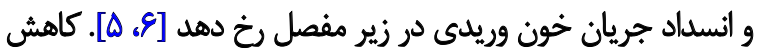

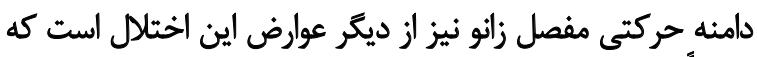

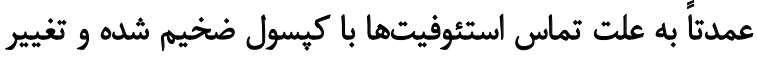

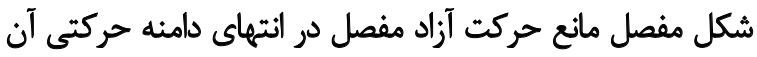

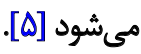

مقدمه

استئوآرتريت' يا بيمارى درثراتيو مفصلى، يكى از شايعترين

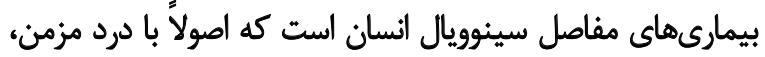
خشكى مفصلى، ضعف عضلى مضلانى، كاهش

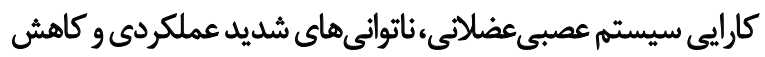

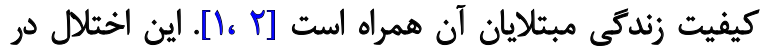

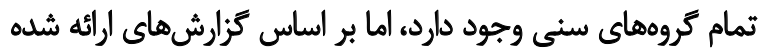

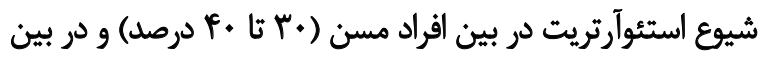

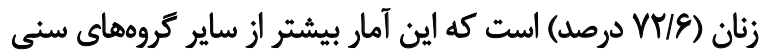

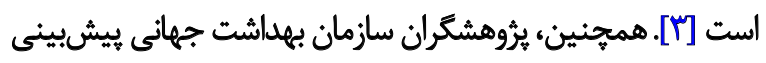




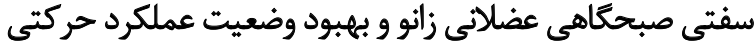

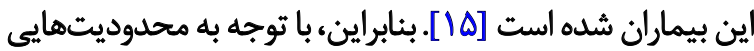

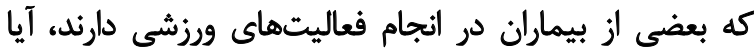

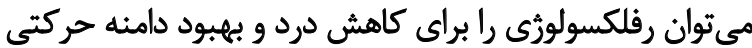

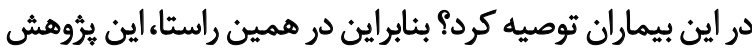

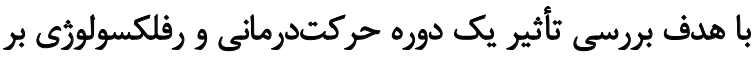

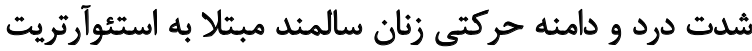

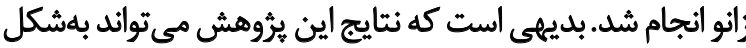

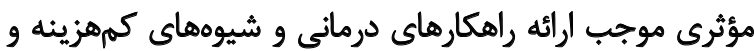

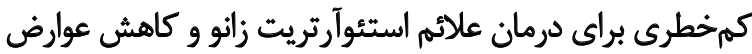
و هزينههاي درمانى آن در بيماران مبتلان باشد.

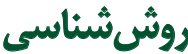

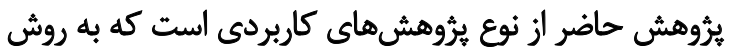

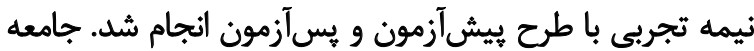

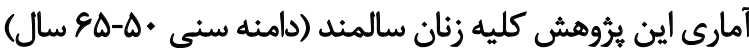

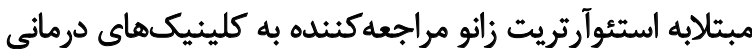

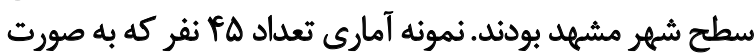

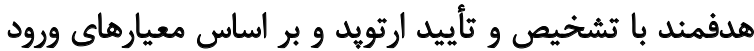

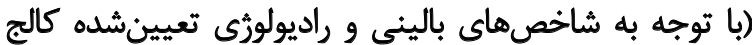

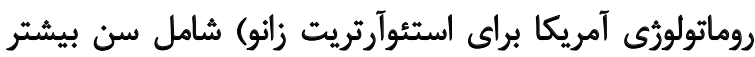

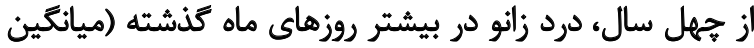

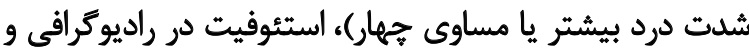

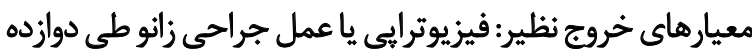

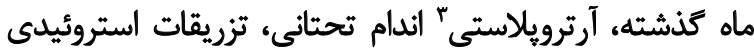

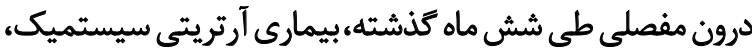

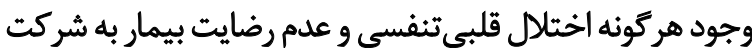

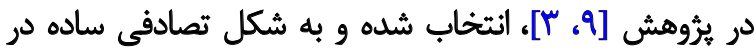
سف تروه يانزدهنفره (حركتدرمانى، رفلكسولوزى و و كنترل) تقسيمبندى شدند.

به منظور اجراي آزمون ابتدا خلاصهاى از برنامههاي درمانى

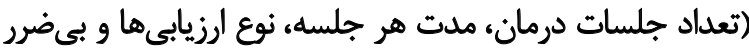

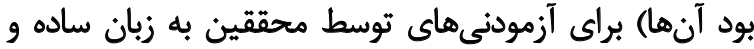

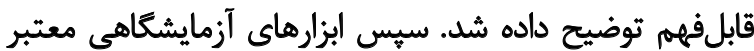

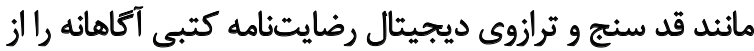

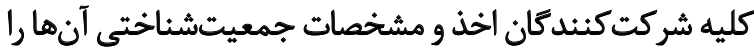

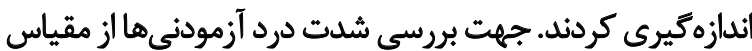

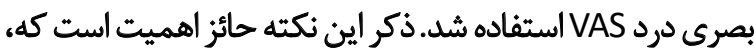

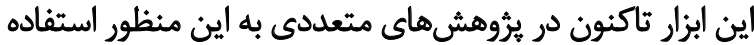

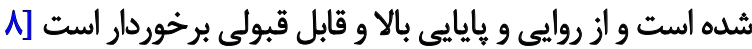

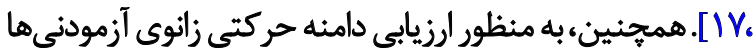
از كَونيامتر مكانيكى فلزى با روش استانداردى كه توسط آكادمى آنيى

3. Arthroplasty

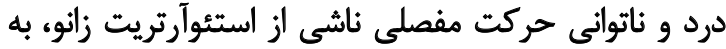

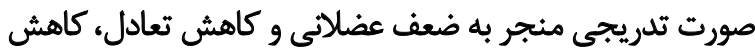
حس عمقى مفصل، تغيير در ياسخ رفلى رفلكسى عضله، كاهش

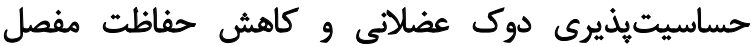

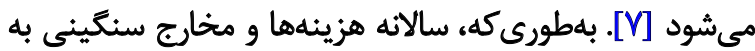

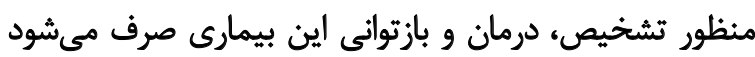

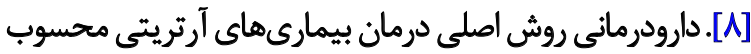

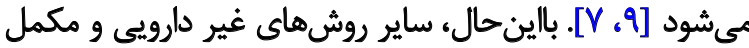

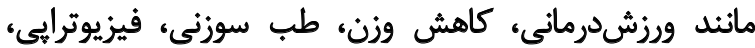

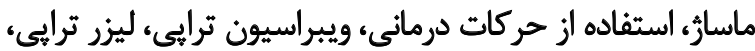

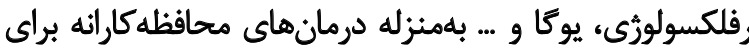

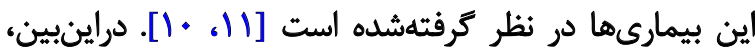

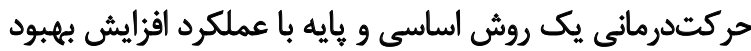

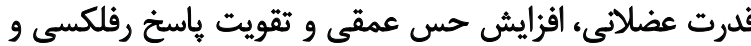

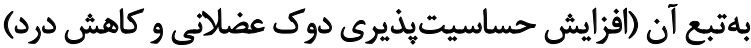

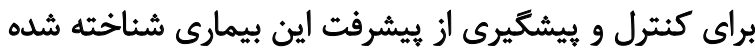

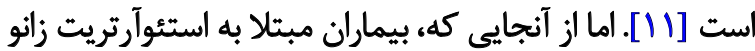

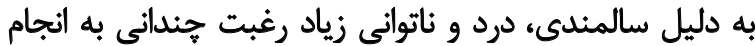

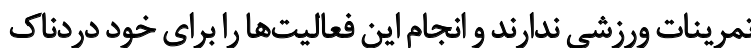

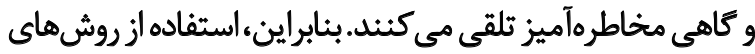

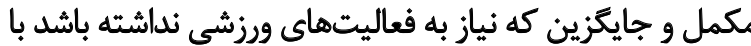

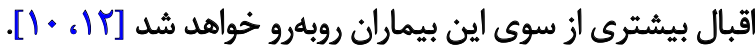

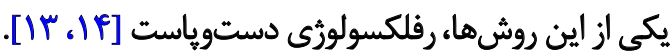
رفلكسولورى يك روش سلامتى كلى نتخر است كه شامل فشار و

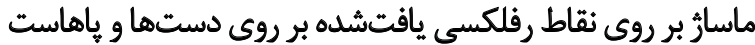

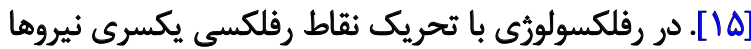

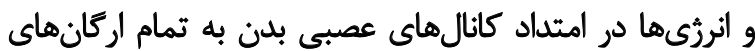

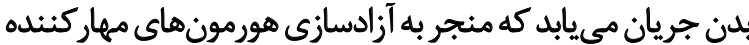

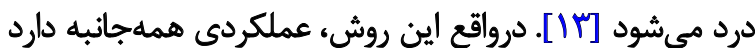

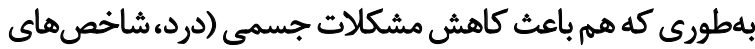

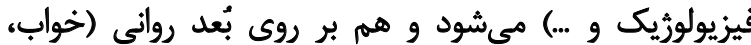

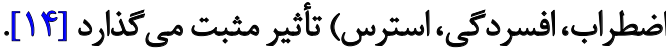

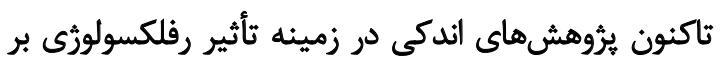

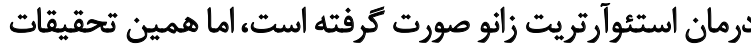

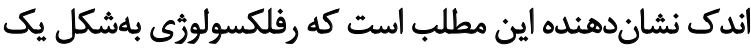

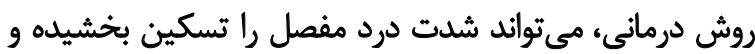

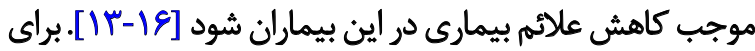

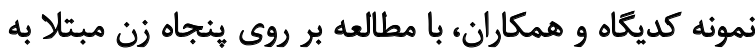

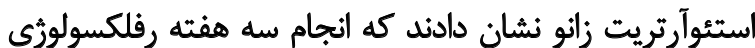

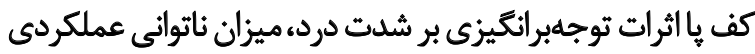

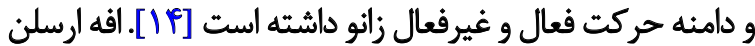

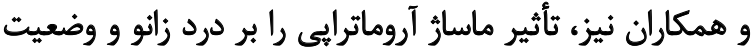

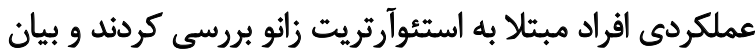

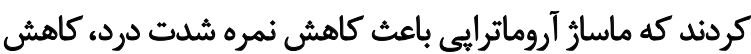




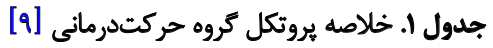

\begin{tabular}{|c|c|c|c|c|c|}
\hline تعداد تكرار & هدف اجراى تمرين & نوع تمرين اجرأُشده & زمان جلسه در روز & طول و مدت دوره & نام مرحله \\
\hline سل ست •"باثيهاي & 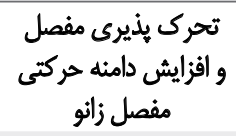 & تمرينات كششى ران و زاثو & ع دقيقه & ئنج جلسه & (مر حله فاز I I I I ) \\
\hline 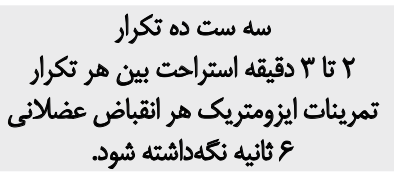 & تقويت عضلات & 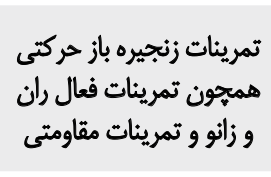 & ع وقيقه & ده جلسه & (مرحله ييش الصيش \\
\hline 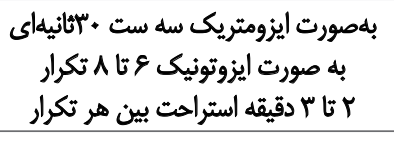 & 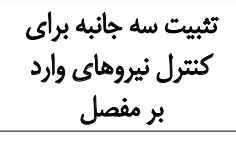 & تمرينات زنجيره بسته & ع دقيقه & ينج جلسه & (مرحله حاد) \\
\hline
\end{tabular}

جدول r. خلاصه هيروتكل Sروه رفلكسولورئ [.r، 19]

\begin{tabular}{|c|c|c|c|c|}
\hline تعداد تكرار در هر جلسه & 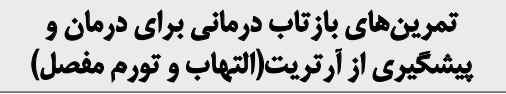 & زمان جلسه در روز & طول و مدت دوره & مرحله \\
\hline 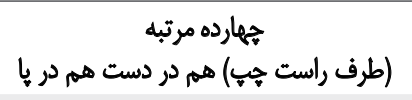 & 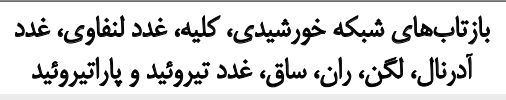 & •r ب دقيقه & ينج جلسه & مقدمائى \\
\hline (طرف راست و جي) همه درد دست هم در يا & 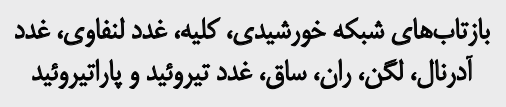 & r برقيقه & يانزيده جلسه & اصلى \\
\hline 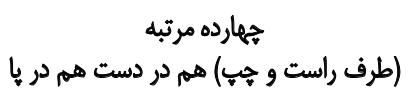 & 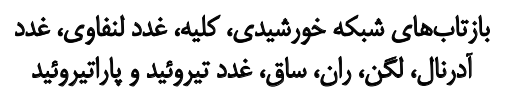 & •ب دقيقه & ينج جلسه & تثبيت \\
\hline
\end{tabular}

نتايج آزمون آمارى شاييرو ويلك در خصوص نرمالبودن دادهها

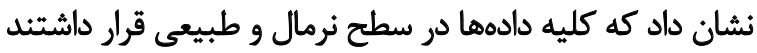

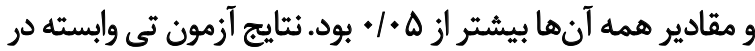

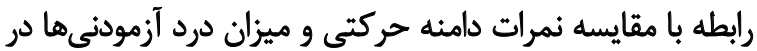

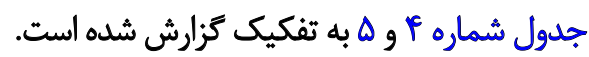
همانطورى كه در جدول شماره f و ه نمايش داده شده است،

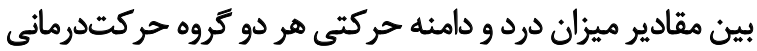

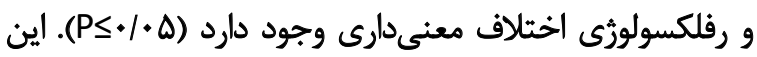

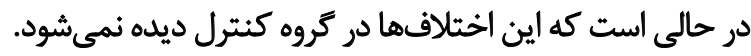

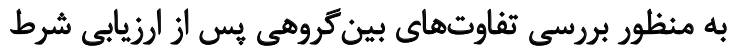

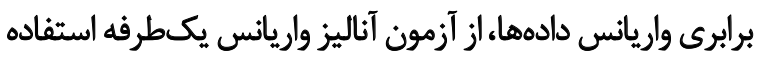

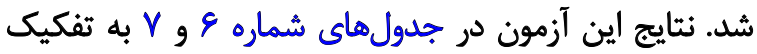
كزارش شده است.

بر اساس نتايج آزمون آناليز واريانس يكطرفه از لحاظ تغييرات

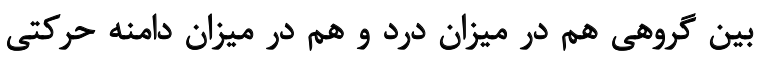

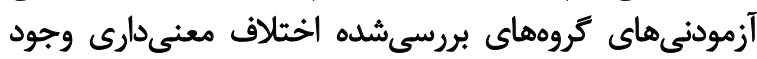

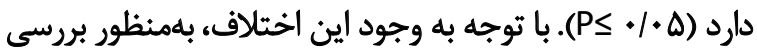

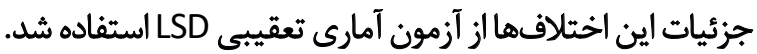

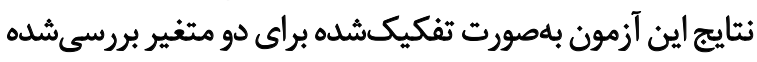

جراحان ارتويدى امريكا ارائهشه، استفاده شد.در اين روش بيمار

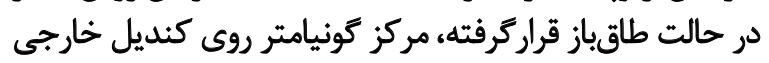

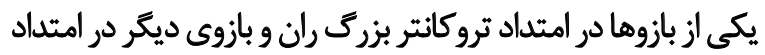

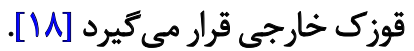

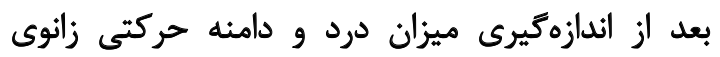

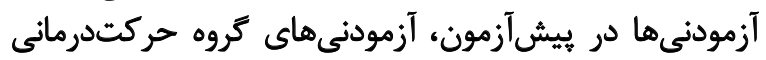

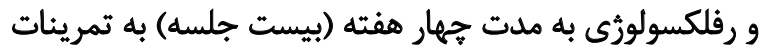

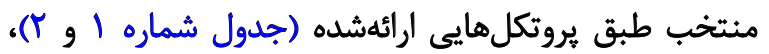

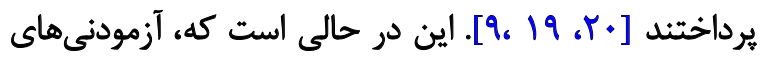

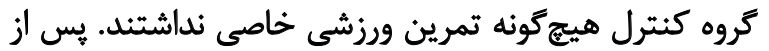

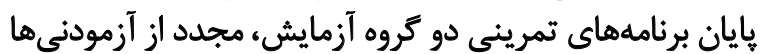

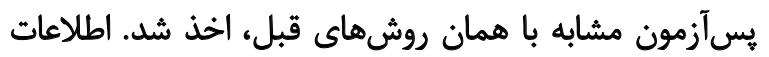

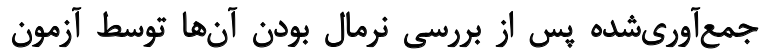

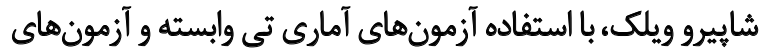

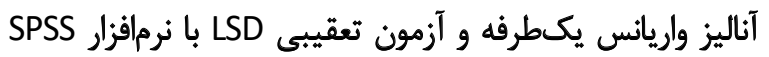

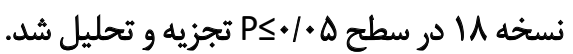
ثنائج در جدولشماره باطلاعات مربوطبهويرَّى هاى جمعيتشناختى

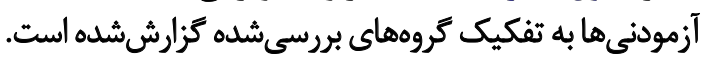


جدول r. ويزٔكى هاى جمعيتشناختى آزمودنىها

\begin{tabular}{|c|c|c|c|c|c|}
\hline \multicolumn{5}{|c|}{ ميانكيندانحراف معيار } & \multirow{2}{*}{ كروهها / متغير } \\
\hline تعداد & (كيلوكرم بر متر') BMI (ك") & وزن (كيلوكرم) & قد (ساتتىمتر) & سن (سال) & \\
\hline يائزده نفر & $r q / r \pm q \Delta / 1 r$ & $V / N \pm \cdot r / r)$ & $\mid \Delta \varphi / N \pm+. / N \Delta$ & $\Delta \Delta / \Delta \pm P / Q / \varphi T$ & حركتدرمانى \\
\hline 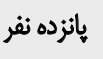 & $Y N I \pm A V / R T$ & $V \Delta / N \pm Y e / p q$ & $181 / \Delta \pm . .11 \mathrm{~A}$ & $\Delta f / \mu \pm . . / \Delta \Delta$ & رفلكسولوزى \\
\hline بانزئد نفر & $r N \backslash \pm R T / R T$ & $\gamma / \mu \pm . \mu / \lambda \Delta$ & LQQ/Y $\pm \& / A F$ & $\Delta S / \Delta \pm q T / \mu T$ & كنترل \\
\hline
\end{tabular}

مجله بيومكانيك ولنش برن

جدول F. نتايج آزمون تى وابسته (مقايسه ميزان درد آزمودنىها)

\begin{tabular}{|c|c|c|c|}
\hline \multirow{3}{*}{$\mathbf{P}$} & \multicolumn{2}{|c|}{ ميانكين+انحراف معيار } & \multirow{3}{*}{ كروهاه / متغير } \\
\hline & \multicolumn{2}{|c|}{ ميزان درد } & \\
\hline & به آزمون & ييش آزمون & \\
\hline $.1+.1 *$ & $r / . . \pm \pm . / 9 Y$ & $r \cdot / T \pm \& / 1$ & حركتّرمانى \\
\hline.$/ . .1 *$ & $r / . \pm \varphi s / M$ & $\Lambda+/ 9 \vee \pm \Phi / *$ & رفلكسولوزى \\
\hline.$\Delta S$ & $N / \Delta \pm . / 91$ & $\varepsilon \cdot|A T \pm \Delta| \cdot$ & كتنرل \\
\hline
\end{tabular}

جدولهـ نتايج آزمون تى وابسته (مقايسه دامنه حركتى آزمودنىها)

\begin{tabular}{|c|c|c|c|}
\hline \multirow{3}{*}{$\mathbf{P}$} & \multicolumn{2}{|c|}{ ميانكيندانحراف معيار } & \multirow{3}{*}{ كروهها / متغير } \\
\hline & \multicolumn{2}{|c|}{ دامنه حركىى } & \\
\hline & يسآزمون & ييش آزمون & \\
\hline$\circ+.1^{\infty}$ & $I T V / r \pm 19 / V \Delta$ & $11 \Delta / r \pm r \Delta / g r$ & حركتدرمانى \\
\hline.$+r^{*}$ & $\| Q / r \pm I N \Delta \Delta$ & $1 . \varphi / 4 \pm 1 . / 10$ & رفلكسولوزى \\
\hline ( & $1.9 / Y \pm .+/ A F$ & $1 \cdot \Delta / r \pm r \Psi / \Delta S$ & كتترل \\
\hline
\end{tabular}

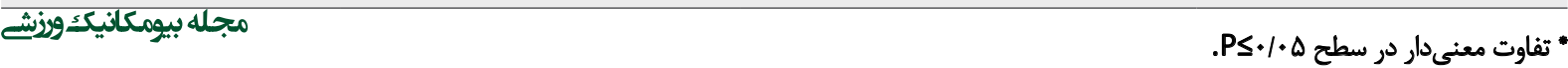

\section{بحث}

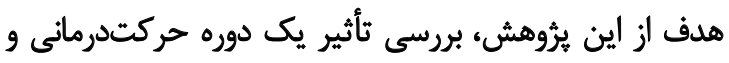

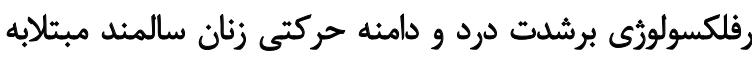

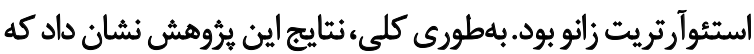

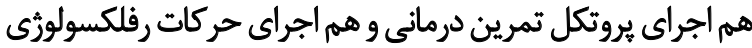

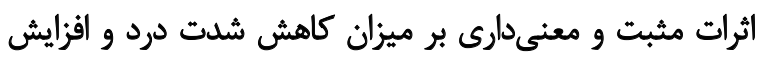

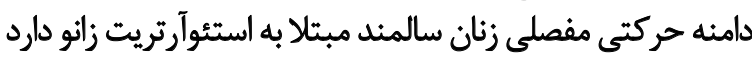
$(P \leq \% \Delta)$

كرجه تاكنون يُوهشى بلصورت خاص و هدفمند به بررسى و

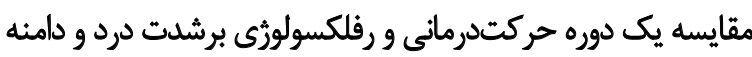

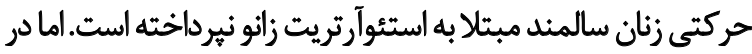

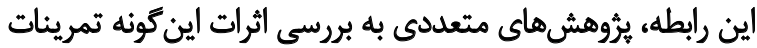

در جدول هاى شماره 1 و 9 كزارش شده است. بر اساس نتايج جدولهاى شماره ^و و 9 بين ميانكين نمرات

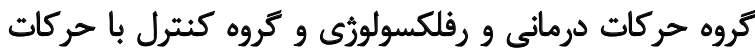

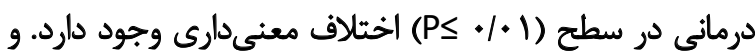

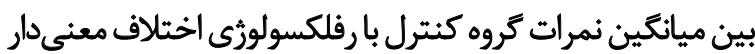

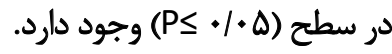

نتايج آزمون تعقيبى LSD نشاندهنده اين مطلب است كه بين

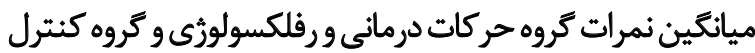

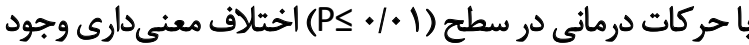

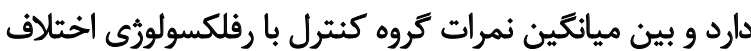

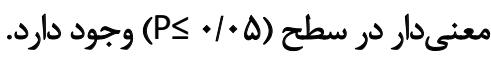


جدول8. نتايج آزمون آناليز واريانس يكسرفه (مقايسه ميزان درد آزمودنىها)

\begin{tabular}{|c|c|c|c|c|c|c|}
\hline $\mathbf{P}$ & $\mathbf{F}$ & ميانكين مربعات & دوجه آزادى & مجموع مربعات & منبع تغييرات & متغير \\
\hline \multirow{3}{*}{$* ..)^{*}$} & \multirow{3}{*}{$\checkmark /|8|$} & l/pr. & $r$ & gl.mf & يين كروهى & \multirow{3}{*}{ ميزان درد } \\
\hline & & $.|| F \mid$ & r & $\Pi / F \Delta S$ & درون كروهى & \\
\hline & & & $P D$ & $0 . / 1 \pi$ & كل & \\
\hline
\end{tabular}

مجله بيومكانيك وزنث

P

جدول V. نتايج آزمون آناليز واريانس يكطرفه (مقايسه ميزان دامنه حركتى آزمودنىها)

\begin{tabular}{|c|c|c|c|c|c|c|}
\hline $\mathbf{P}$ & $\mathbf{F}$ & ميانكين مربعات & درجه أزادى & مجموع مربعاث & منبع تغييرات & متغير \\
\hline \multirow{3}{*}{ •/.M"** } & \multirow{3}{*}{$11 / \mathrm{rgV}$} & $1 / 11$ & $r$ & $1 \mathrm{~W} \cdot \mathrm{Mr}$ & بين كروهى & \multirow{3}{*}{ دامنه حركتى } \\
\hline & & I/MTY & pro & TNAEN & درون كروهي & \\
\hline & & & Fo & sV/IVA & كل & \\
\hline
\end{tabular}

مجله بيومكانيك ورنث

مكانيزم رفلكسولوزي بر كاهش شدت درد مشخص نشده است.

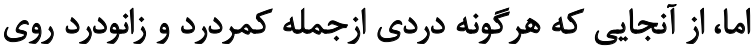

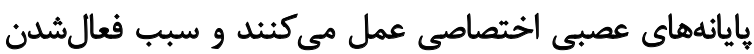

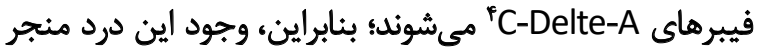
به افزايش ترشح هورمونهاى استرسزا، تحريك غده فوق كليه

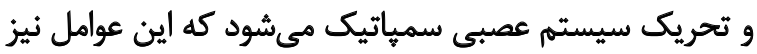

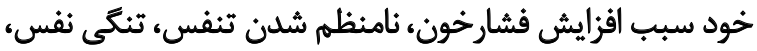

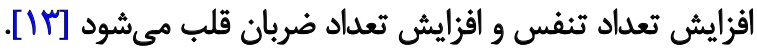
درنتيجه، برخى محققان در ثوجيه اثرات رفلكسولوزئى بر بر ميزان

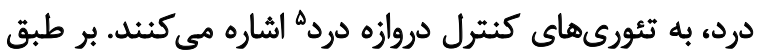

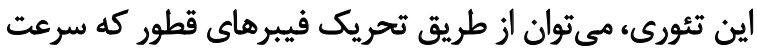

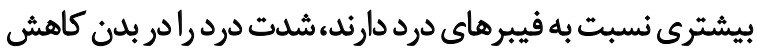

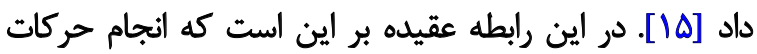

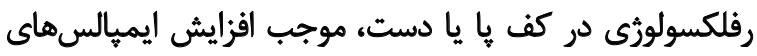

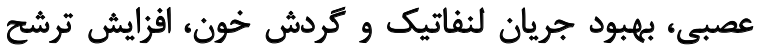
اندورفينها و انكفالينها، بهببود دفع سموم بدن، افزايش تقويت

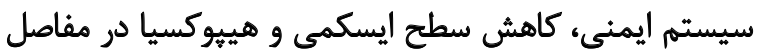

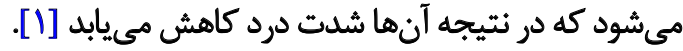
ناظمزاده و همكاران در اين راسثا نشان دادند كه، انجام

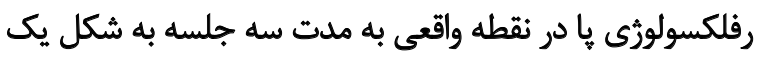

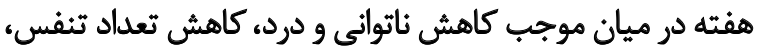

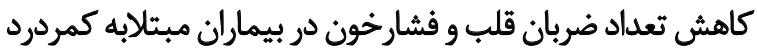

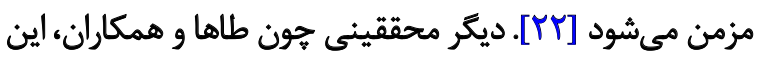

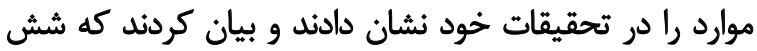

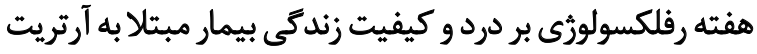

4. Deleting c fibers

5. Gate control theory of pain

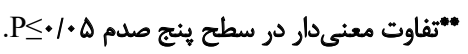

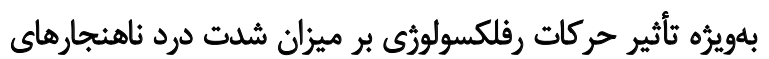

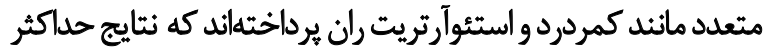

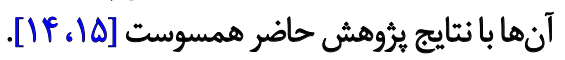

براي نمونه در اين زمينه، كديڤاه و همكاران، با مطالعه بر روى

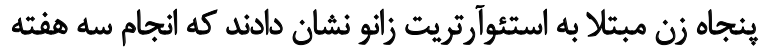

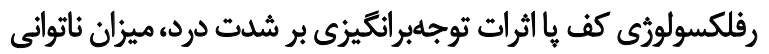

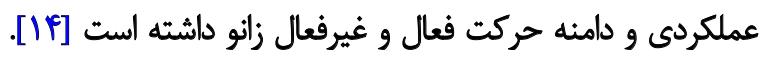

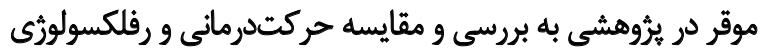

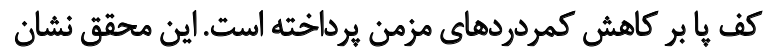

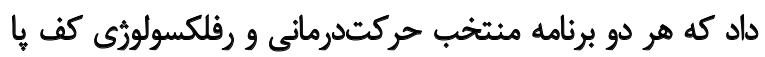
موجب كاهش معنى دارى بر ميزان دردهاي كمر شده است. كه به به

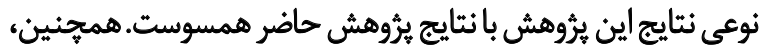

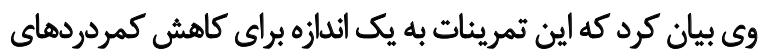

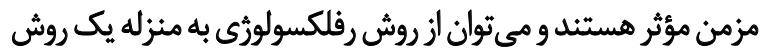

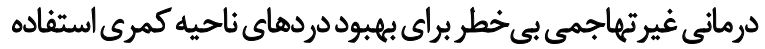

كرد [10]

كايلرو همكاران، در مطالعه خود به بررسى اثرات حركات

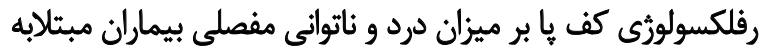
استئوآرتريت مفصل ران برداختند.اين محققين نشان دادند كه انجام

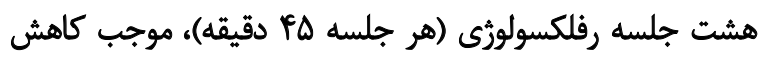

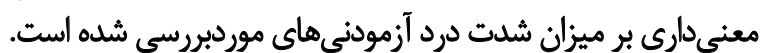

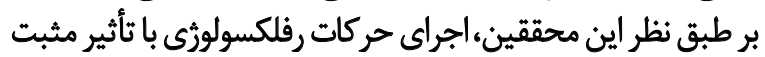

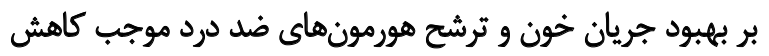
ميزان درد، رفع ناتوائى مفصلي اين بيمارن تردان و بهبود كيفيت خواب

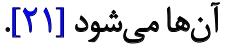

كرجه، هنوز به صورت دقيق و قطعى جكونكي عملكرد و 
جدول A. نتايج آزمون تعقيبى LSD كروهاى موردبررسى در ميزان درد

\begin{tabular}{|c|c|c|c|c|}
\hline $\mathbf{P}$ & خطاى اتحراف از ميانكين & تفاوت ميانكينها & \multicolumn{2}{|c|}{ منبع تغيير } \\
\hline.$\% I^{*}$ & $r / \pi$ & re/Nr & كروه رفلكسولوزى & \multirow{2}{*}{ كروه كترل } \\
\hline$\%$ & (E & TNES & كروه حركتدرمانى & \\
\hline.. $.1 *$ & Q/99 & $M T / P Q$ & كروه رفلكسولوزى & كروه حركتدرمانى \\
\hline
\end{tabular}

جدول 9. نتايج آزمون تعقيبي LSD كروهاي موردبررسي در ميزان دامئه حركتي

\begin{tabular}{|c|c|c|c|c|}
\hline $\mathbf{P}$ & خطاى انحراف از ميانكين & تفاوت ميانكينها & \multicolumn{2}{|c|}{ منبع تغيير } \\
\hline$\cdot / \cdot H^{*}$ & $r /+r$ & $r q / F a$ & كروه رفلكسولوزى & كروه كنتل \\
\hline$+1+.+* *$ & P/TA & TW/EV & كروه حركتدرمائى & \\
\hline$+1+\infty)^{* *}$ & $\varepsilon / * V$ & $81 / 40$ & كروه رفلكسولوزى & كروه حركتدرمانى \\
\hline
\end{tabular}

مجله بيومكانيك ولشث

خاطر تأثير و استفاده مستقيم تمرينات كششى، تمرينات زنجيره

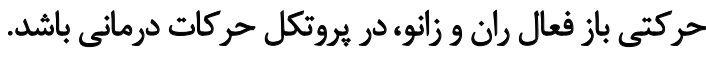
از سوى ديكر، جون وجود درد علاوه بر احساس ناراحتى در بيماران

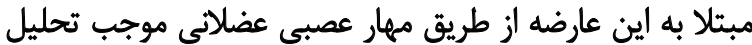

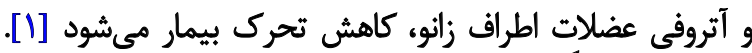

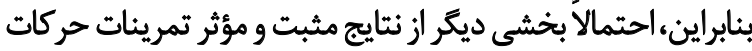

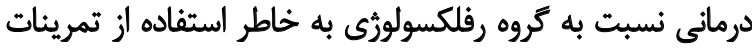

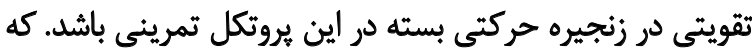

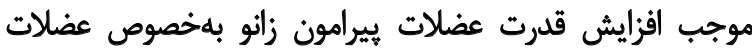

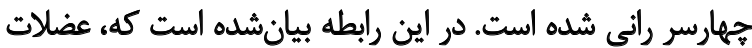

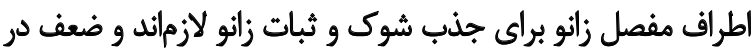

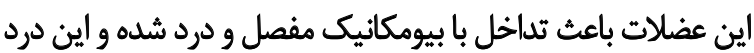

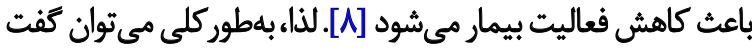

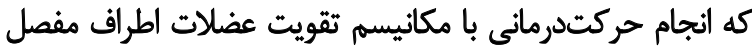

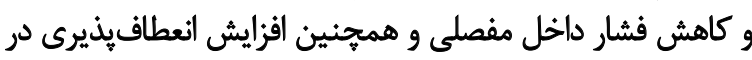

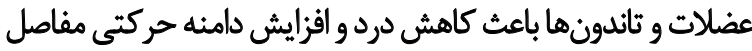

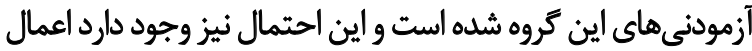

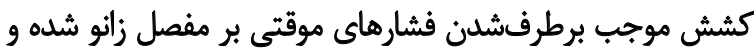

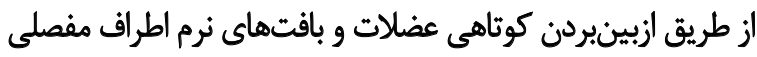

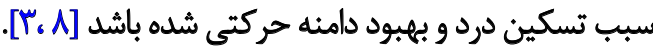
از ديكر عوامل مؤثر در ميزان درد و دامنه حركتى افراد مبتلا

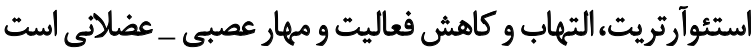

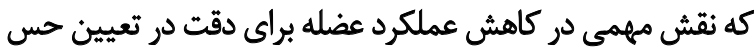

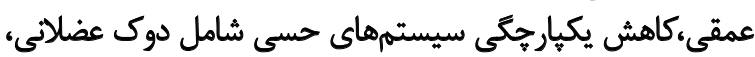

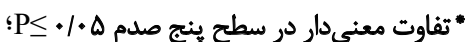

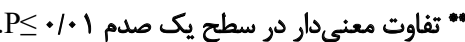
روماتوئيد اثر مثبت و معنى دارى دارد [YY].

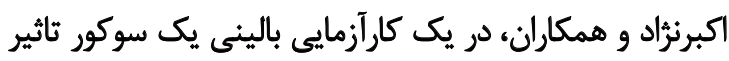

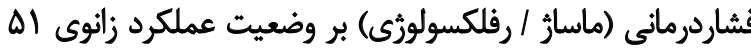

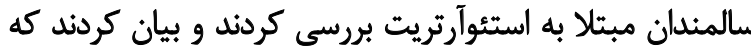

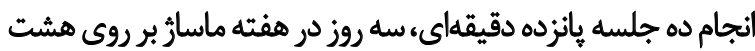

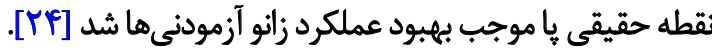

همجنين، متين و همكاران، تأثير ماساز آروماترايى و و

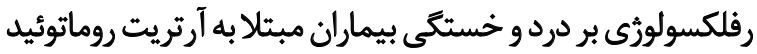

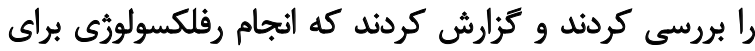

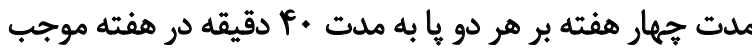

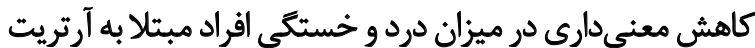

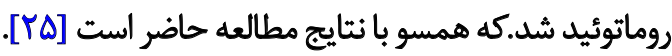

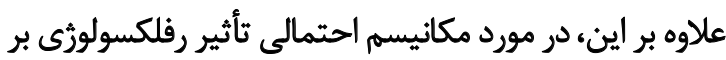

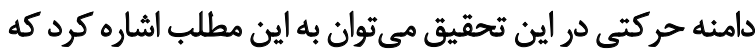

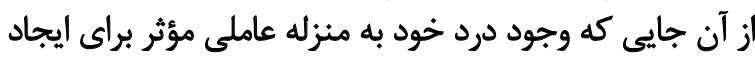

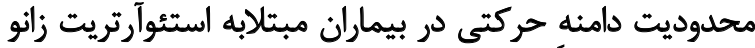

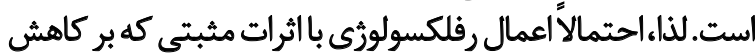

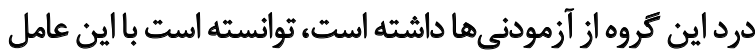

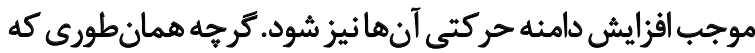

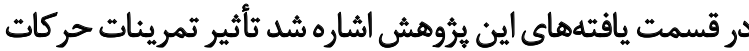

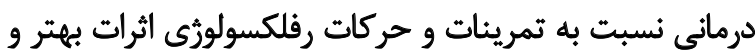

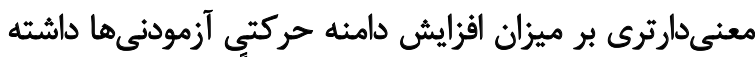

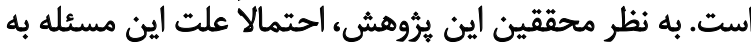




\section{ملاحظات اخلاقى \\ ييروى از اصول اخلاق يثوهش}

اين يُروهش از لحاظ رعايت موارد اخلاقي، يس از تاييد نظر

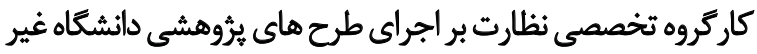

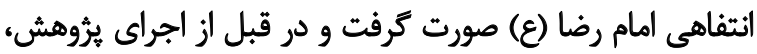

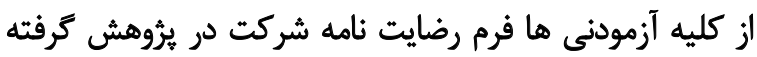

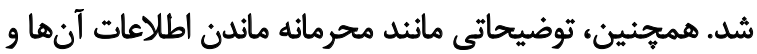

احتيارى بودن خروج از برؤهش به آزمودنى ها ارائه شد بودانه

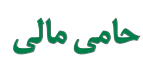

اين مقاله بركرفته شده از إياين نامه كارشناسى ارشد نويسنده

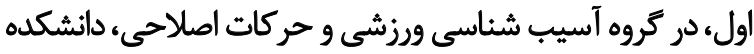

علوم انساني، دانشكاه بين المللى امام رضا (ع)، مشهد است.

$$
\text { مشاركت نويسند انمان }
$$

تمام نويسندكان در طراحى، اجرا و نكارش همه بخشهاى ئزوهش حاضر مشاركت داشتهاند.

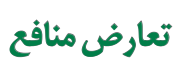

بنابر اظهار نويسندكان، اين مقاله تعارض منافع ندارد.
كاهش سيستم بينايى و وستيبولار و تغيير در حساسيت كيرندههاى

جلدى و مفصلى مي شود [IV].

بر اساس نتايج تحقيقات انجام شده عملكرد مناسب مكانيسم

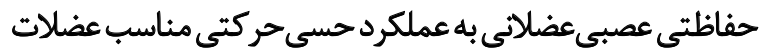
اطراف مفصل بستكى دارد. اختلال در عملكرد حستى حسى عضى مضلات

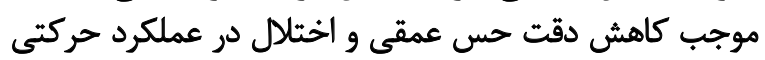

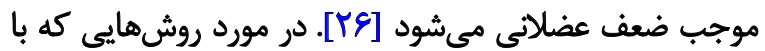

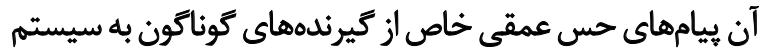

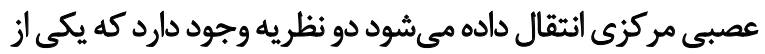

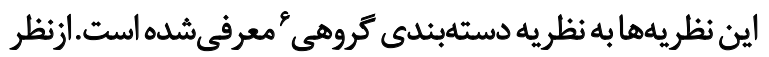

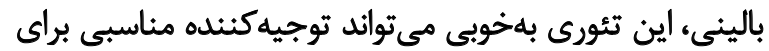

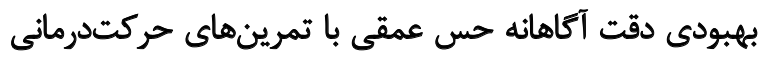

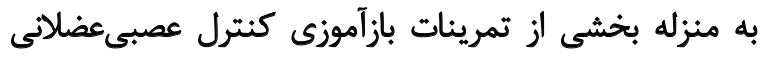

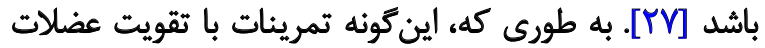

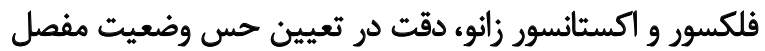

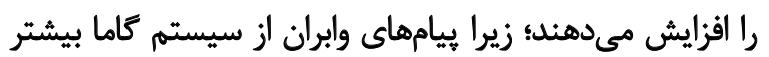

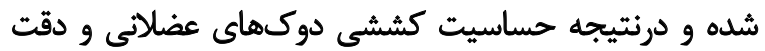

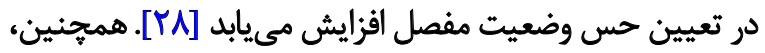

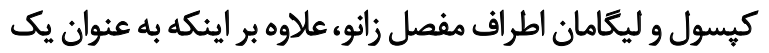

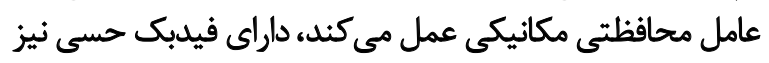

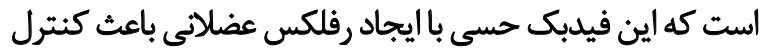

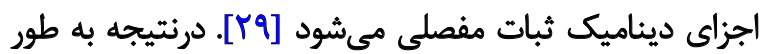

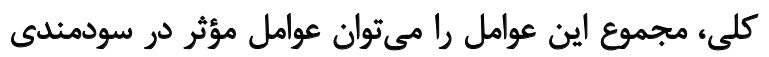

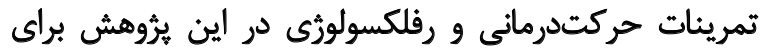
بيماران مبتلا به اين عارضه، اشاره كرد.

\section{تثيجه كيرى نهايى}

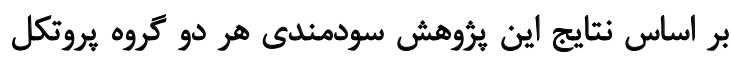

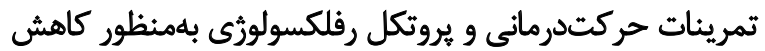

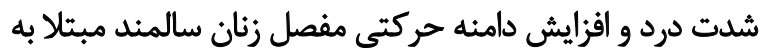

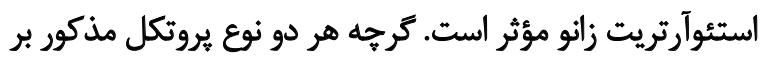

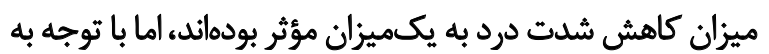

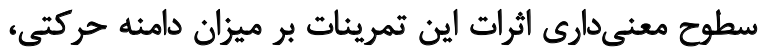

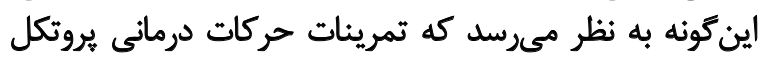

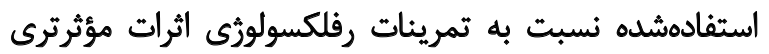

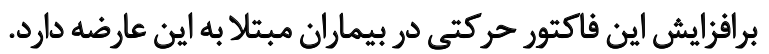

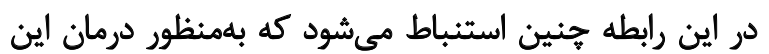

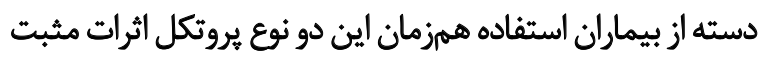

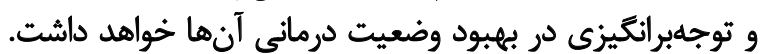

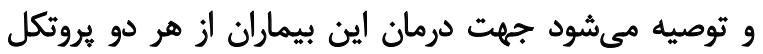
حركتدرمانى و رفلكسولوزى استفاده شود. 


\section{References}

[1] Alkhawajah HA, Alshami AM. The effect of mobilization with movement on pain and function in patients with knee osteoarthritis: $A$ randomized double-blind controlled trial. BMC Musculoskelet Disord. 2019; 20(1):452. [DOI:10.1186/s12891-019-2841-4] [PMID] [PMCID]

[2] Alfredo PP, Bjordal JM, Dreyer SH, Meneses SR, Zaguetti G, Ovanessian $\mathrm{V}$, et al. Efficacy of low level laser therapy associated with exercises in knee osteoarthritis: A randomized double-blind study. Clin Rehabil. 2012; 26(6):523-33. [DOI:10.1177/0269215511425962] [PMID]

[3] Rastgar Koutenaei F, Mosallanezhad Z, Naghikhani M, Biglarian A, Nouroozi M, Ghodrati M. [The effect of low level laser therapy on pain and range of motion of patients with knee osteoarthritis (Persian)]. PTJ. 2017; 7(1):13-8. [DOI:10.29252/nrip.ptj.7.1.13]

[4] Johnson VL, Hunter DJ. The epidemiology of osteoarthritis. Best Pract Res Clin Rheumatol. 2014; 28(1):5-15. [DOI:10.1016/j. berh.2014.01.004] [PMID]

[5] Peat G, McCarney R, Croft P. Knee pain and osteoarthritis in older adults: A review of community burden and current use of primary health care. Ann Rheum Dis. 2001; 60(2):91-7. [DOI:10.1136/ ard.60.2.91] [PMID] [PMCID]

[6] Alenazi AM, Alshehri MM, Alothman S, Alqahtani BA, Rucker J, Sharma N,et al. The Association of Diabetes with Knee pain Severity and Distribution in people with Knee osteoarthritis using Data from the osteoarthritis initiative. Sci Rep. 2020; 10(1):1-8. [DOI:10.1038/ s41598-020-60989-1] [PMID] [PMCID]

[7] Brooks PM. Impact of osteoarthritis on individuals and society: how much disability? Social consequences and health economic implications. Curr Opin Rheumatol. 2002; 14(5):573-577 [DOI:10.1097/00002281-200209000-00017] [PMID]

[8] Sartipzadeh M, Moazami M, Mohammadi MR. [The effect of core stabilization training on elderly balance and knee pain with knee osteoarthritis (Persian)]. J Paramed Sci Rehab. 2016; 5(3):7-17. http:// jpsr.mums.ac.ir/article_7342.html

[9] Ezadpanah A, Moazami M, Khoshraftar Yazdi N. [Effect of a period of therapeutic exercise and detraining after that on balance in the women with knee osteoarthritis (Persian)]. J ModRehabil. 2016; 9 (S1):101-109. http://mrj.tums.ac.ir/article-1-5405-en.html

[10] Abbasi E, Kahrizi S, Razi M, Faghihzadeh S. [The effects of whole body vibration therapy on pain, range of motion and functional activities in patients with osteoarthritis of the knee (Persian)]. J Med Counc Iran. 2016; 34(2):125-37. https://www.sid.ir/en/journal/ViewPaper.aspx?id=551097

[11] Aghajani A. [The effect of eight weeks selected exercises on balance indexes in elderly patients with knee osteoarthritis (Persian)]. J Paramed Sci Rehab. 2017; 6(2):86-97. http://jpsr.mums.ac.ir/article $8726 . h t m l$

[12] Verschueren SM, Roelants M, Delecluse C, Swinnen S, Vanderschueren D, Boonen S. Effect of 6-month whole body vibration training on hip density, muscle strength, and postural control in postmenopausal women: A randomized controlled pilot study. J Bone Miner ReS. 2004; 19(3):352-359. [DOI:10.1359/JBMR.0301245] [PMID]

[13] Nisha V. Effectiveness of foot reflexology on pain among patients with osteoarthritis in selected villages at Tirunelveli. [PhD. dissertation]. Chennai: Sri K. Ramachandran Naidu College of Nursing; 2014. https://www.semanticscholar.org/paper/Effectiveness-of-footreflexology-on-pain-among-in-Nisha/c72dc142b9c937dff9f6a1ea4025f846e6bcedd4
[14] Abdelaziz KhS, Botla AM, Ebrahim HH, Gab AA. Ebrahim and Amir A. Effect of reflexology on knee osteoarthritis patients: A randomized clinical trial. Gab World JSport Sci. 2019; 14(1):21-27. https://idosi. org/wjss/14(1)19/4.pdf

[15] Movaghar MH. [The comparison of exercise therapy and foot reflexology on reducing chronic low back pain (Persian)]. J North Khorasan Univ Med Sci. 2012; 4(4):701-706. [DOI:10.29252/jnkums.4.4.701]

[16] Arslan DE, Kutlutürkan S, Korkmaz M. The effect of aromatherapy massage on knee pain and functional status in participants with osteoarthritis. Pain Management Nursing. 2019; 20(1):62-9. [DOI:10.1016/j.pmn.2017.12.001] [PMID]

[17] Shah Hosseini GR, Negahban Siuki H, Madani SA, Ebrahimi Takamjani E, Shaterzadeh MJ. [Comparison of the effect of two therapeutic methods (traditional \& new) on therapeutic parameters in patients with primary knee osteoarthritis (Persian)]. Razi J Med Sci. 2004 10(37):735-41. http://rjms.iums.ac.ir/article-1-222-en.html

[18] Mazloum V, Rahnama N, Khayambashi K. [Effects of therapeutic exercise and hydrotherapy on pain severity and knee range of motion in patients with hemophilia: A randomized controlled trial (Persian)]. Int J Prev Med. 2014; 5(1):83-6. [PMID] [PMCID]

[19] Sadeghi M, Zabolipour S, Afrasiabifar A, Najafi Doulatabad S. [Comparison of the Effect of Sole Reflexology Massage and Stretching Exercises on Pain Severity of Patients with Rheumatoid Arthritis (Persian)]. J Clinic Care Skill. 2020; 1(3):103-7. http://jccs.yums.ac.ir/ article-1-79-en.html

[20] Ricks S. The reflexology workout: Hand \& foot massage for super health \& rejuvenation. New York: Crow Trade Publication; 1995.

[21] Kielar K, Drzał-Grabiec J, Truszczyńska A, Twarowska N. The effects of reflexology on joint pain and disability in patients with osteoarthritis of the hip joints. Postepy Rehabilitacji. 2017; 31(3):29-40. [DOI:10.1515/rehab-2015-0071]

[22] Taha NM, Ali ZH. Effect of reflexology on pain and quality of life in a patient with rheumatoid arthritis. Marsland Press. 2011 8:357-65. http://www.publications.zu.edu.eg/Pages/PubShow. aspx?|D=1981\&\&pub|D=1

[23] Zadeh MN, Rezvani M, Jalalodini A, Navidian A, Yosefian N, Ghalje $M$, et al. [The effect of reflexology massage on physiological parameters in patients with chronic low back pain (Persian)]. Pajoohandeh J. 2013; 17(6):286-90. http://pajoohande.sbmu.ac.ir/article-1-1415-en. html

[24] Akbarnezhad N. [The effect of acupressure on physical function of knee among elderly in elderly patients with osteoarthritis: A randomized clinical trial (Persian)]. Nurs J Vulnerable. 2016; 2(5):1-14. https://www.sid.ir/en/journal/ViewPaper.aspx?ID=556094

[25] Metin ZG, Ozdemir L. The effects of aromatherapy massage and reflexology on pain and fatigue in patients with rheumatoid arthritis: A randomized controlled trial. Pain Management Nursing. 2016; 17(2):140-9. [DOI:10.1016/j.pmn.2016.01.004] [PMID]

[26] Farzaneh $\mathrm{H}$, Ilbeigi S, Anbarian M. [The effects of fatigue on rear foot angle in normal and pronate individuals (Persian)]. J ParamedSci Rehab. 2016; 5(3):83-92. https://www.sid.ir/en/Journal/ViewPaper. aspx?ID=568018

[27] Goodman M, Marks R. The association between knee proprioception and isotonic quadriceps femoris strength. Physiotherapy Canada. 1998; 50(1):53-7. https://www.researchgate.net/publication/292747553 
[28] Riemann BL, Lephart SM. The sensorimotor system, part I: the physiologic basis of functional joint stability. J Athl Train. 2002; 37(1):71-9. [PMCID] [PMID]

[29] Hassan BS, Mockett S, Doherty M. Static postural sway, proprioception, and maximal voluntary quadriceps contraction in patients with knee osteoarthritis and normal control subjects. Ann Rheum Dis. 2001; 60(6):612-8. [DOI:10.1136/ard.60.6.612] [PMID] [PMCID] 